\title{
EARLY-TIME VLA OBSERVATIONS AND BROADBAND AFTERGLOW ANALYSIS OF THE FERMI/LAT DETECTED GRB 130907A
}

\author{
Péter Veres $^{1,2}$, Alessandra Corsi ${ }^{3}$, Dale A. Frail ${ }^{4}$, S. Bradley Cenko ${ }^{5,6}$, And Daniel A. Perley ${ }^{7}$ \\ ${ }^{1}$ The George Washington University, Department of Physics, 725 21st St., NW, Washington, DC 20052, USA; peter.veres@uah.edu \\ ${ }^{2}$ Center for Space Plasma and Aeronomic Research (CSPAR), University of Alabama in Huntsville, Huntsville, AL 35899, USA \\ Texas Tech University, Department of Physics, Box 41051, Lubbock, TX 79409-1051, USA \\ ${ }^{4}$ National Radio Astronomy Observatory, P.O. Box O, Socorro, NM 87801, USA \\ 5 Astrophysics Science Division, NASA Goddard Space Flight Center, Mail Code 661, Greenbelt, MD 20771, USA \\ 6 Joint Space-Science Institute, University of Maryland, College Park, MD 20742, USA \\ ${ }^{7}$ Department of Astronomy, California Institute of Technology, MC 249-17, 1200 East California Blvd., Pasadena, CA 91125, USA \\ Received 2014 November 26; accepted 2015 July 20; published 2015 August 26
}

\begin{abstract}
We present multi-wavelength observations of the hyper-energetic gamma-ray burst (GRB) 130907A, a Swiftdiscovered burst with early radio observations starting at $\approx 4 \mathrm{hr}$ after the $\gamma$-ray trigger. GRB 130907A was also detected by the Fermi/LAT instrument and at late times showed a strong spectral evolution in X-rays. We focus on the early-time radio observations, especially at $>10 \mathrm{GHz}$, to attempt to identify reverse shock signatures. While our radio follow-up of GRB 130907A ranks among the earliest observations of a GRB with the Karl G. Jansky Very Large Array, we did not see an unambiguous signature of a reverse shock. While a model with both reverse and forward shock can correctly describe the observations, the data is not constraining enough to decide upon the presence of the reverse-shock component. We model the broadband data using a simple forward-shock synchrotron scenario with a transition from a wind environment to a constant density interstellar medium (ISM) in order to account for the observed features. Within the confines of this model, we also derive the underlying physical parameters of the fireball, which are within typical ranges except for the wind density parameter $\left(A_{*}\right)$, which is higher than those for bursts with wind-ISM transition, but typical for the general population of bursts. We note the importance of early-time radio observations of the afterglow (and of well-sampled light curves) for unambiguously identifying the potential contribution of the reverse shock.
\end{abstract}

Key words: gamma-ray burst: general

\section{INTRODUCTION}

Gamma-ray bursts' (GRBs) afterglows still pose some fundamental unanswered questions. The processes giving rise to prompt and early-afterglow emission at optical and radio frequencies are among the least well understood. Early-time optical (Akerlof et al. 1999) and radio (Kulkarni et al. 1999) flashes were first discovered in GRB 990123 and attributed to reverse-shock emission (e.g., Mészáros \& Rees 1999; Sari \& Piran 1999; Corsi et al. 2005; Urata et al. 2014). But later on, fast robotic telescopes did not find evidence for early optical flashes in the expected numbers (Melandri et al. 2008). It has been suggested that the lack of early optical emission may be due to the fact that the reverse shock peaks below the optical range, at millimeter or centimeter wavelengths (Kulkarni et al. 1999; Chandra \& Frail 2012; Laskar et al. 2013a; Perley et al. 2014). Another possibility is that the reverse shock is entirely suppressed by, e.g., a high degree of magnetization of the ejecta (Zhang \& Kobayashi 2005).

Here we present early-time radio observations of GRB 130907A, together with observations at other wavelengths. Our radio follow-up of GRB 130907A ranks among the earliest observations of a GRB with the Very Large Array (VLA). However, our data do not show a clear reverse shock signature. Besides an early-time radio followup and a self-absorbed radio spectrum, the other interesting features of this burst consist of an early-time Fermi/LAT detection and a significant late-time spectral evolution in the $\mathrm{X}$-ray band.

Our paper is organized as follows. In Section 2 we present the observational data for this burst and discuss the spectral and temporal properties of GRB 130907A. In Section 3 we provide a theoretical interpretation for the broadband data, and conclude in Section 4. In this paper, we use the $F_{\nu} \propto t^{-\alpha} \nu^{-\beta}$ notation ( $\alpha$ is the temporal index and $\beta$ is the spectral index) and $Q=10^{x} Q_{x}$ for any physical quantity $Q$ in cgs units (unless otherwise stated).

\section{OBSERVATIONS AND DATA ANALYSIS}

\subsection{Gamma-Rays}

GRB 130907A (Page et al. 2013) was discovered by the BAT instrument on board the Swift satellite (Gehrels et al. 2004) at 21:41:13 UT. It was also detected by the Fermi/LAT (Vianello et al. 2013), Konus WIND (Golenetskii et al. 2013), and various ground-based observatories at longer wavelengths (e.g., Corsi 2013; Gorbovskoy et al. 2013a). With a redshift of $z=1.238$, this GRB occurred at a luminosity distance of $D_{\mathrm{L}}=2.7 \times 10^{28} \mathrm{~cm}$ (de Ugarte Postigo et al. 2013), calculated using the following cosmological parameters: $\Omega_{m}=0.27, \Omega_{\Lambda}=0.73$, and $H_{0}=73 \mathrm{~km} \mathrm{~s}^{-1} \mathrm{Mpc}^{-1}$. The isotropically emitted energy is $E_{\text {iso }} \sim 3.0 \times 10^{54} \mathrm{erg}$ (calculated from $1 \mathrm{keV}$ to $10 \mathrm{MeV}$ in the local frame). The jet opening angle $\left(\theta_{j} \gtrsim 12^{\circ}\right.$, see Section 3.4$)$ corrected energy is $E_{\text {jet }} \gtrsim$ $3 \times 10^{52} \mathrm{erg}$, which makes this burst part of the hyper-energetic class of GRBs (Cenko et al. 2011). Moreover, at $18 \mathrm{ks}$ after the $\gamma$ ray trigger, the Fermi/LAT detected one of the highest-energy $(55 \mathrm{GeV})$ photons ever observed in a GRB (Vianello et al. 2013). We refer the reader to Tang et al. (2014) for more details about the LAT flux measurements. 


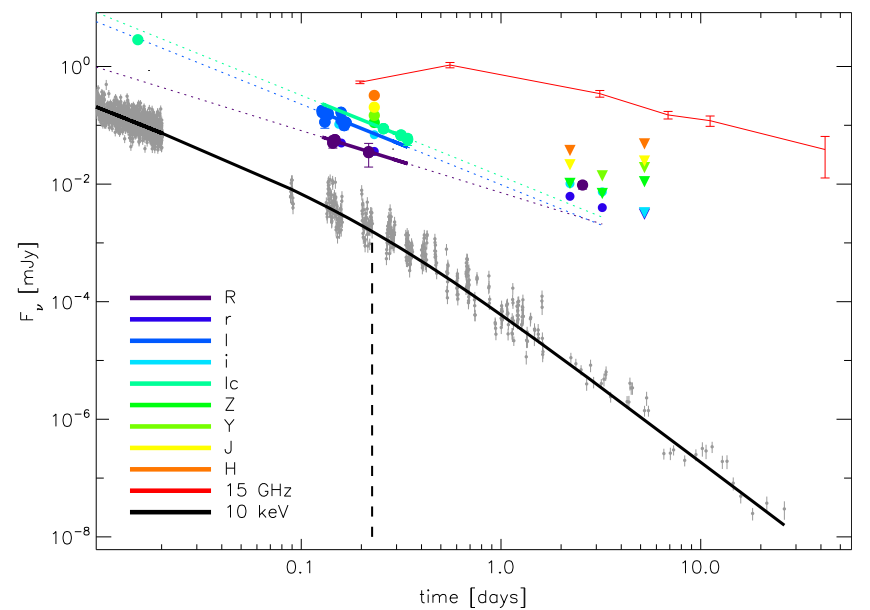

Figure 1. Optical, radio, and X-ray flux measurements of GRB 130907A. The fit to the optical data is only for the intervals marked with thick continuous lines; the dotted lines are extrapolations. Triangles mark upper limits. The vertical line marks the temporal break in the X-ray fit.

\subsection{X-Rays}

X-ray measurements by Swift/XRT of GRB 130907A started at $\approx 56 \mathrm{~s}$ after the trigger (during the burst prompt $\gamma$ ray emission phase) and lasted until $\approx 26$ day after the burst. The light curve is overall declining, with an easily identifiable break around 0.2 days since the trigger (Figure 1) and a strong spectral evolution at late times (Figure 2). Due to this spectral evolution, we used a dynamic count-to-flux density conversion and derived an accurate $10 \mathrm{keV}$ light curve using the burst analyser (Evans et al. 2007, 2010).

The $10 \mathrm{keV}$ light curve of GRB 130907A shows a clear break followed by a steepening of the temporal decay index (Figure 1). In what follows, for our analysis of the afterglow, we discard all observations at $t<300 \mathrm{~s}$ due to a possible contribution of the prompt emission. By fitting the X-ray light curve with a smoothly broken power law of the form $F_{\nu}=A\left[\left(t / t_{\text {break }}\right)^{\alpha_{1}}+\left(t / t_{\text {break }}\right)^{\alpha_{2}}\right]^{-1}$ (Beuermann et al. 1999) we find $t_{\text {break }}=0.23 \pm 0.02$ days and indices: $\alpha_{1}=1.32 \pm$ 0.02 and $\alpha_{2}=2.57 \pm 0.05$, respectively before and after the break. We did not attempt to find the parameter responsible for the smoothness of the break and fixed it to the nominal value of 1 (the $s$ parameter in Beuermann et al. 1999). This could tentatively explain why the fit underestimated the points close to the break.

We have obtained the spectral data from the XRT repository's spectral tools. ${ }^{8}$ The spectral index before the temporal break is unusually hard: $\beta_{\mathrm{X} \text {,early }}=0.69 \pm 0.06$ with no significant evolution. A unique feature of the X-ray afterglow is the spectral evolution, starting with the light curve break, from $\beta_{\mathrm{X}}=0.8 \pm 0.1$ at early times to $\beta_{\mathrm{X}}=1.7 \pm 0.4$ at later times (see Figure 2). A linear fit (in $\log t-\beta$ ) to the first three points gives a slope of $0.32 \pm 0.58$, consistent with no spectral evolution, while for the last four points the slope is $0.52 \pm 0.16$, indicative of an evolving spectral index. The average spectral index after the break is $\beta_{\mathrm{X} \text {,late }}=0.96 \pm 0.05$. The X-ray absorbing column of the host galaxy is: $N_{H}=(9.8 \pm$ 1.1) $\times 10^{21} \mathrm{~cm}^{-2}$.

We finally note that the light curve integrated for the entire energy interval of XRT $(0.3-10 \mathrm{keV})$ has a different behavior

\footnotetext{
http://www.swift.ac.uk/xrt_spectra
}

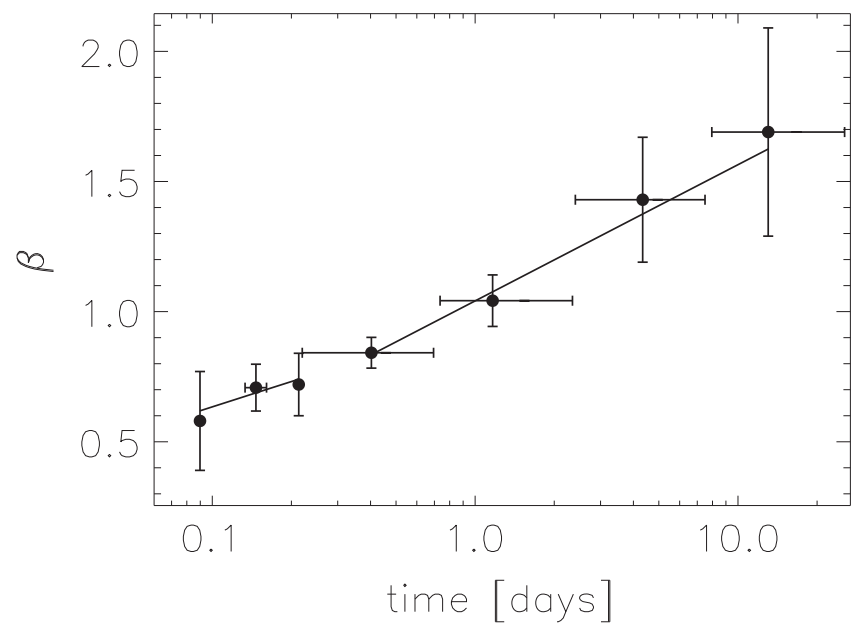

Figure 2. Evolution of the X-ray spectral index with time. Spectral indices were obtained using the XRT spectral repository (see Section 2.2).

than the flux density light curve plotted in Figure 1. Indeed, the automatic fitting routine (Evans et al 2009) yields a broken power-law fit with three breaks for the integrated light curve (see http://www.swift.ac.uk/xrt_live_cat/00569992/). On the other hand, a fit with a smoothly broken power-law function (similar to the one we fitted to the flux density light curve) does not constrain the break time, while a simple power-law fit gives a slope of $\alpha=1.510 \pm 0.003$. The latter is in strong contrast with the steep slope found for the late-time flux density (Figure 1), and it is indicative of a changing X-ray spectrum at late times.

\subsection{Optical}

We gathered the optical observations of GRB 130907A from the public GCN bulletins (see Table 1). In the case of Skynet observations, where the data points were reported on figures, we obtained the numerical flux values by digitizing these figures.

We correct the optical data of GRB 130907A for Galactic absorption $\left(A_{V}=0.03 \mathrm{mag}\right)$ in the direction of the burst using the maps of Schlafly \& Finkbeiner (2011). We find that the optical spectral index at the time of the first radio observation is $\approx 2.5$, suggesting that the host galaxy strongly absorbs the optical flux. We fit the I, Ic, and R filter measurements from 0.12 to 0.35 days to obtain the temporal decay index for this time interval (Figure 1), and we found $\alpha_{\mathrm{I}}=1.37 \pm 0.38$ (from 7 measurements; Table 1), $\alpha_{\text {Ic }}=1.38 \pm 0.59$ (from 3 measurements; Table 1), and $\alpha_{\mathrm{R}}=1.05 \pm 1.06$ (from 3 measurements; Table 1). The other optical observations, at earlier and later times, are too sparse to derive secure spectral or temporal information. We note, however, that late-time measurements seem to lie above the extrapolation from the temporal decay derived between 0.12 and 0.35 days, thus suggesting a late-time flattening (Figure 1). A foreground galaxy (SDSS J142333.95+453626.2 with photometric redshift $z=0.6 \pm 0.3$ ) at a distance of $\approx 0$ ". 5 from GRB $130907 \mathrm{~A}$ has been identified by Lee et al. (2013), with $i$ and $r$ magnitudes comparable to that of the afterglow at $\approx 3$ days after the trigger. This galaxy could tentatively explain the flattening of the optical afterglow at these late times. On the other hand, Butler et al. (2013c) have limited the contribution of galaxy flux in the $r$-and $i$-bands to $>22.6 \mathrm{mag}$. 
Table 1

Optical Observations of GRB 130907A

\begin{tabular}{|c|c|c|c|c|}
\hline Time $_{\text {mid }}$ (days) & Filter & $\operatorname{mag}(\mathrm{AB})$ & Instrument & References \\
\hline $5 \times 10^{-4}$ & $\mathrm{r}$ & $15 \pm(0.2)$ & MASTER & (1) \\
\hline 0.0152 & Ic & $15.26 \pm 0.03$ & Tautenburg & (2) \\
\hline 0.0172 & $\mathrm{Rc}$ & $16.57 \pm 0.03$ & Tautenburg & (2) \\
\hline 0.127 & I & $18.27 \pm 0.15$ & Skynet & (3) \\
\hline 0.131 & $\mathrm{I}$ & $18.76 \pm 0.23$ & Skynet & (3) \\
\hline 0.132 & I & $18.41 \pm 0.084$ & Skynet & (4) \\
\hline 0.136 & $\mathrm{I}$ & $18.40 \pm 0.12$ & Skynet & (3) \\
\hline 0.144 & $\mathrm{R}$ & $19.65 \pm 0.20$ & Skynet & (3) \\
\hline 0.148 & $\mathrm{R}$ & $19.51 \pm 0.13$ & Skynet & (4) \\
\hline 0.154 & $\mathrm{i}^{\prime}$ & $18.86 \pm 0.09$ & Skynet & (4) \\
\hline 0.158 & I & $18.35 \pm 0.13$ & Skynet & (3) \\
\hline 0.159 & $\mathrm{r}^{\prime}$ & $19.65 \pm 0.10$ & Skynet & (4) \\
\hline 0.164 & I & $18.91 \pm 0.16$ & Skynet & (3) \\
\hline 0.167 & $\mathrm{I}$ & $18.79 \pm 0.09$ & Skynet & (4) \\
\hline 0.217 & $\mathrm{R}$ & $20.06 \pm 0.47$ & $\mathrm{~T} 21$ & (5) \\
\hline 0.232 & $\mathrm{r}$ & $20.01 \pm 0.03$ & RATIR & (6) \\
\hline 0.232 & $\mathrm{i}$ & $19.30 \pm 0.02$ & RATIR & (6) \\
\hline 0.258 & Ic & $19.05 \pm 0.09$ & Skynet & (4) \\
\hline 0.287 & $\mathrm{Rc}$ & $19.91 \pm 0.11$ & Skynet & (4) \\
\hline 0.316 & Ic & $19.31 \pm 0.13$ & Skynet & (4) \\
\hline 0.341 & Ic & $19.51 \pm 0.22$ & Skynet & (4) \\
\hline 2.22 & $\mathrm{r}$ & $21.92 \pm 0.12$ & RATIR & (7) \\
\hline 2.22 & $\mathrm{i}$ & $21.38 \pm 0.09$ & RATIR & (7) \\
\hline 2.56 & $\mathrm{R}$ & $21.44 \pm 0.16$ & Maidanak & (8) \\
\hline 3.22 & $\mathrm{r}$ & $22.40 \pm 0.14$ & RATIR & (9) \\
\hline 3.22 & $\mathrm{i}$ & $21.73 \pm 0.10$ & RATIR & (9) \\
\hline $8.56 \times 10^{-4}$ & white & $15.45 \pm 0.02$ & UVOT & (10) \\
\hline $7.13 \times 10^{-3}$ & v & $16.29 \pm 0.16$ & UVOT & (10) \\
\hline $6.27 \times 10^{-3}$ & $\mathrm{~b}$ & $16.78 \pm 0.12$ & UVOT & (10) \\
\hline $3.31 \times 10^{-3}$ & $\mathrm{u}$ & $15.87 \pm 0.04$ & UVOT & (10) \\
\hline $7.71 \times 10^{-3}$ & w1 & $18.54 \pm 0.31$ & UVOT & (10) \\
\hline $7.42 \times 10^{-3}$ & $\mathrm{~m} 2$ & $>19.20$ & UVOT & (10) \\
\hline $6.85 \times 10^{-3}$ & w2 & $>18.70$ & UVOT & (10) \\
\hline 0.232 & $\mathrm{Z}$ & $18.78 \pm 0.05$ & RATIR & (6) \\
\hline 0.232 & Y & $18.48 \pm 0.06$ & RATIR & (6) \\
\hline 0.232 & $\mathrm{~J}$ & $18.13 \pm 0.06$ & RATIR & (6) \\
\hline 0.232 & $\mathrm{H}$ & $17.63 \pm 0.05$ & RATIR & (6) \\
\hline 2.22 & $\mathrm{Z}$ & $>21.37$ & RATIR & (7) \\
\hline 2.22 & $\mathrm{Y}$ & $>20.87$ & RATIR & (7) \\
\hline 2.22 & $\mathrm{~J}$ & $>20.58$ & RATIR & (7) \\
\hline 2.22 & $\mathrm{H}$ & $>19.97$ & RATIR & (7) \\
\hline 3.22 & $\mathrm{Z}$ & $>21.80$ & RATIR & (7) \\
\hline 3.22 & $\mathrm{Y}$ & $>21.05$ & RATIR & $(7)$ \\
\hline 5.23 & $\mathrm{r}$ & $>22.68$ & RATIR & (11) \\
\hline 5.23 & $\mathrm{i}$ & $>22.62$ & RATIR & (11) \\
\hline 5.23 & $\mathrm{Z}$ & $>21.30$ & RATIR & (11) \\
\hline 5.23 & $\mathrm{Y}$ & $>20.70$ & RATIR & (11) \\
\hline 5.23 & $\mathrm{~J}$ & $>20.42$ & RATIR & (11) \\
\hline 5.23 & $\mathrm{H}$ & $>19.69$ & RATIR & (11) \\
\hline
\end{tabular}

References. (1) Gorbovskoy et al. (2013b); (2) Schmidl et al. (2013); (3) Trotter et al. (2013a); (4) Trotter et al. (2013b); (5) Hentunen et al. (2013); (6) Lee et al. (2013); (7) Butler et al. (2013a); (8) Pozanenko et al. (2013); (9) Butler et al. (2013b); (10) Oates \& Page (2013); and (11) Butler et al. (2013c).

To account for the extinction (reddening) in the host galaxy, we use the absorption curves of Gordon et al. (2003). The relation between the absorbed and unabsorbed flux is: $F_{\nu}^{\text {obs }}=F_{\nu}^{\text {unabs }} \times 10^{-0.4 A(\nu)}$. Here $A(\nu)$ is the absorption curve, characterized by a $V$-band value, $A_{V}$, which is a free parameter, and a shape which is usually taken to be similar to the LMC, SMC, or the Milky Way. Due to the small number of optical

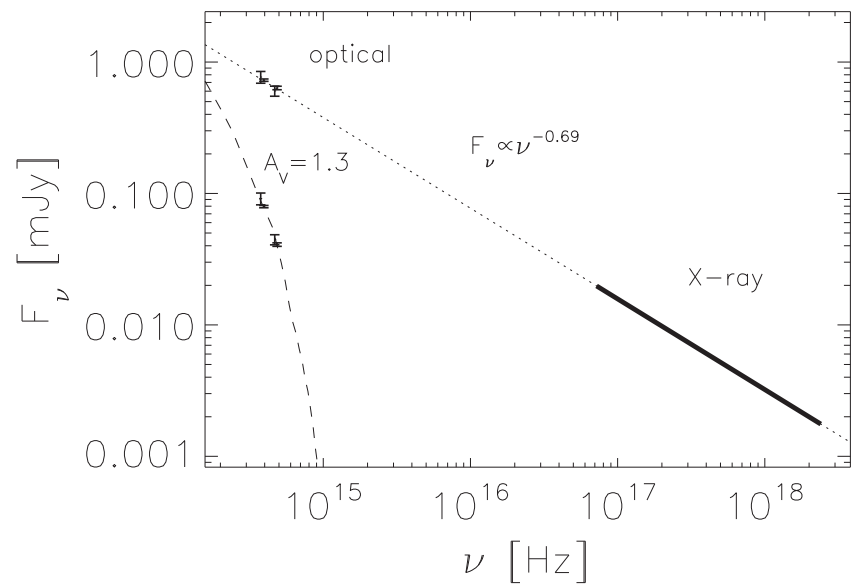

Figure 3. Illustration of the host galaxy extinction on the optical measurements at Epoch I. The thick line represents the measured X-ray spectrum; the dotted line is its extrapolation. Dashed lines show the absorbed spectral energy distribution that fits the observations (measurements atop the dashed curve) The points on the dotted line are the de-absorbed data points.

observations, we assume the SMC extinction curve, which is most commonly used for GRB afterglow studies (Schady et al. 2010). We have the best spectral coverage in the optical band around the time of Epoch $\mathrm{I}(t=0.193$ days $)$, where we have extrapolated the available filters ( $\mathrm{R}, \mathrm{Rc}, \mathrm{I}, \mathrm{Ic})$ from $\sim 0.1$ to $\sim 0.4$ days. By further assuming that the optical and $\mathrm{X}$-ray spectral indices are the same $(\beta=0.69$; at this early time there is no significant spectral evolution in X-rays, and the optical and X-ray temporal slopes are consistent with being the same within the large uncertainties) we find $A_{V} \approx 1.3 \pm 0.1$ in the frame of the host galaxy (see Figure 3).

The above value of $A_{V}$ is derived by fitting a power law with the same spectral index as the X-ray measurements to the unabsorbed optical points. Indeed, we can exclude a spectral break between the optical and X-ray regimes: if there was a break, the optical spectral index would be $\beta=-1 / 3$, which, after correcting for extinction, would imply a true optical flux that is incompatible with the X-ray data.

Using the X-ray spectral analysis described in Section 2.2, we estimate $N_{H} / A_{V} \approx 7.5 \times 10^{21} \mathrm{~cm}^{-2}$. This value is on the lower side, but is still consistent with the $N_{H} / A_{V}$ distribution reported in Schady et al. (2010). Generally speaking, GRB host galaxies have systematically larger $N_{H} / A_{V}$ ratios compared to the Magellanic clouds and the Milky Way (Schady et al. 2010), and this effect is at least partly intrinsic to the host galaxies. Bearing in mind the uncertainties on $A_{V}$ due to the small number of optical measurements, the $N_{H} / A_{V}$ value of the host galaxy of GRB 130907A is more like the SMC than most GRB sightlines.

\subsection{Radio}

Radio observations of GRB 130907A were performed with the VLA $^{9}$ (Perley et al. 2009) in its CnB and B configurations, under our Target of Opportunity programs. ${ }^{10}$

Our observations of GRB 130907A rank among the earliest VLA detections of a GRB (see Figure 4). The follow-up started

\footnotetext{
9 The National Radio Astronomy Observatory is a facility of the National Science Foundation operated under cooperative agreement by Associated Universities, Inc.; http://www.nrao.edu/index.php/about/facilities/vlaevla

${ }^{10}$ VLA/14A-430-PI: A. Corsi; VLA/S50386-PI: S.B.Cenko.
} 

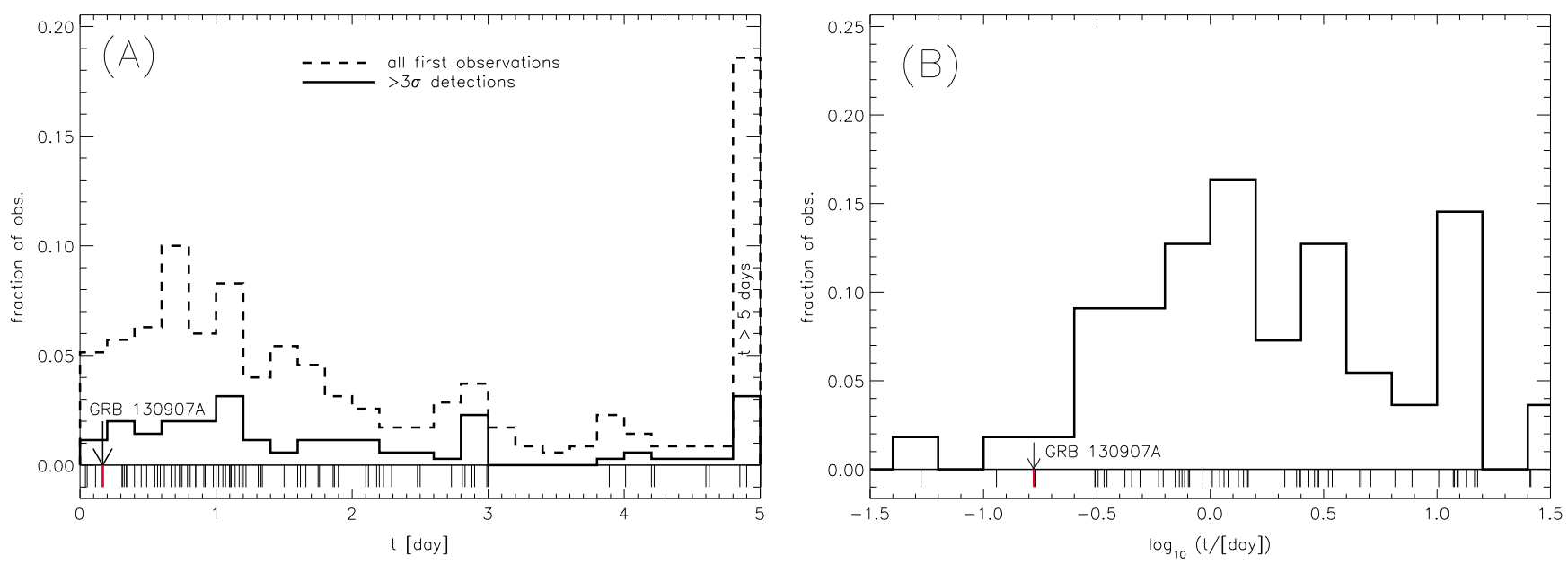

Figure 4. (A): Distribution of time delays from the $\gamma$-ray trigger to the first radio observation of GRBs from GRB 970111 to GRB 141109A. The histogram represents the earliest observation for a given burst. The plot shows the detections $(>3 \sigma)$ out of 350 observations. The bulk of the observations were carried out with the VLA, and later with the Karl G. Jansky VLA. Other observatories include: ATCA, the Ryle telescope, AMI, WSRT, JCMT, MAMBO, OVRO, and CARMA. The rug shows time delays for the detections and the arrow indicates the time delay to the first radio observation/detection of GRB 130907A. (B): Histogram of the first detections (above $3 \sigma$ ) at frequencies greater than $10 \mathrm{GHz}$. The total number of bursts detected at frequencies above $10 \mathrm{GHz}$ is 55 . Delay times for bursts before GRB 110731A are compiled in Chandra \& Frail (2012). For the others we have used: GRB 141109A: Corsi (2014a); GRB 141026A: Corsi (2014b); GRB 140903A: Fong (2014); GRB 140713A: Zauderer et al. (2014a); GRB 140709A: Anderson et al. (2014); GRB 140703A: Corsi (2014c); GRB 140515A: Laskar et al. (2014b); GRB 140419A: Perley (2014); GRB 140311A: Tanvir et al. (2014) and Laskar et al. (2014a); GRB 140304A: Zauderer et al. (2014b); GRB 131224A: Fong et al. (2013d); GRB 131108A: Perley (2013c); GRB 130912A: Fong et al. (2013c); GRB 130907A: Corsi (2013); GRB 130822A: Fong et al. (2013b); GRB 130702A: Corsi et al. (2013); and Perley \& Kasliwal (2013); GRB 130609A: Zauderer et al. (2013c); GRB 130606A: Laskar et al. (2013b); GRB 130603B: Fong et al. (2013a); GRB 130518A:Castro-Tirado et al. (2013); GRB 130427A: Zauderer et al. (2013a) and Perley (2013b); GRB 130418A: Perley (2013a); GRB 130215A: Perley \& Keating (2013); GRB 130131A: Zauderer et al. (2013b); GRB 121226A: Fong et al. (2012b); GRB 121024A: Laskar et al. (2012c); GRB 120923A: Zauderer et al. (2012a); GRB 120804A: Fong et al. (2012a); GRB 120802A: Laskar et al. (2012b); GRB 120729A: Laskar et al. (2012a); GRB 120521C: Zauderer \& Berger (2012b); GRB 120404A: Zauderer et al. (2012b); GRB 120327A: Hancock et al. (2012); GRB 120326A: Perley et al. (2012); GRB 120305A: Zauderer et al. (2012c); GRB 120119A: Zauderer \& Berger (2012a); GRB 111215A: Horesh et al. (2011); GRB 111209A: Hancock et al. (2011); GRB 111117A: Fong et al. (2011); GRB 111022B: Zauderer \& Berger (2011); GRB 111008A: Zauderer et al. (2011); and- GRB 111005A: Michalowski et al. (2011).

at $3.64 \mathrm{hr}$ after the trigger. Hereafter, we refer to the first VLA observation as Epoch I or EI; later VLA observations are labeled incrementally up to Epoch $\mathrm{V}$ at 42 days after the trigger (Table 2).

VLA data were reduced and imaged using the Common Astronomy Software Applications package. Specifically, the calibration was performed using the VLA calibration pipeline v1.2.0. After running the pipeline, we inspected the data (calibrators and target source) and applied further flagging when needed. 3C286 and J1423+4802 were used as flux and phase calibrators, respectively. The VLA measurement errors are a combination of the rms map error, which measures the contribution of small unresolved fluctuations in the background emission and random map fluctuations due to receiver noise, and a basic fractional error (here conservatively estimated to be $\approx 5 \%$, based on the flux variations measured for the phase calibrator; Ofek et al. 2011) that accounts for inaccuracies of the flux density calibration. These errors were added in quadrature and the total errors are reported in Table 2. An additional source of error in the radio band can occur from scintillation, which we discuss in Section 3.5.

We also observed the position of GRB 130907A with the Combined Array for Research in Millimeter Astronomy (CARMA) on 2013 September 08 between 21:46:36 and 23:12:35 UT $\left(t_{\mathrm{mid}}=1.03\right.$ day). Observations were conducted in single-polarization mode with the $3 \mathrm{~mm}$ receivers tuned to a frequency of $93 \mathrm{GHz}$, and were reduced using the Multichannel Image Reconstruction, Image Analysis and Display environment (MIRIAD). Flux calibration was established by a short observation of Mars at the beginning of the track. We detect no
Table 2

Radio Observations of GRB 130907A

\begin{tabular}{lcccc}
\hline \hline Time $_{\text {mid }}($ days $)$ & $\nu(\mathrm{GHz})$ & Flux $(\mu \mathrm{Jy})$ & Instrument & References \\
\hline $0.193(\mathrm{EI})$ & 19.2 & $630 \pm 25$ & EVLA & (this work) \\
$0.193(\mathrm{EI})$ & 24.5 & $1160 \pm 28$ & EVLA & (this work) \\
0.550 & 15 & $1060 \pm 110$ & AMI & (1) \\
1.03 & 93 & $<1100(2 \sigma)$ & CARMA & (this work) \\
1.735 & 5 & $190 \pm 30$ & WSRT & (2) \\
$3.08(\mathrm{EII})$ & 8.5 & $441 \pm 34$ & EVLA & (this work) \\
$3.08(\mathrm{EII})$ & 11 & $444 \pm 45$ & EVLA & (this work) \\
$3.08(\mathrm{EII})$ & 13.5 & $347 \pm 24$ & EVLA & (this work) \\
$3.08(\mathrm{EII})$ & 16 & $358 \pm 43$ & EVLA & (this work) \\
$6.86(\mathrm{EIII})$ & 5 & $204 \pm 22$ & EVLA & (this work) \\
$6.86(\mathrm{EIII})$ & 7.4 & $93 \pm 21$ & EVLA & (this work) \\
$6.86(\mathrm{EIII})$ & 8.5 & $208 \pm 16$ & EVLA & (this work) \\
$6.86(\mathrm{EIII})$ & 11 & $152 \pm 20$ & EVLA & (this work) \\
$11.15(\mathrm{EIV})$ & 13.5 & $155 \pm 24$ & EVLA & (this work) \\
$11.15(\mathrm{EIV})$ & 16 & $109 \pm 21$ & EVLA & (this work) \\
$41.96(\mathrm{EV})$ & 5 & $62 \pm 12$ & EVLA & (this work) \\
$41.96(\mathrm{EV})$ & 7.4 & $45 \pm 10$ & EVLA & (this work) \\
$41.96(\mathrm{EV})$ & 8.5 & $61 \pm 26$ & EVLA & (this work) \\
$41.96(\mathrm{EV})$ & 11 & $72 \pm 19$ & EVLA & (this work) \\
\hline
\end{tabular}

Note. Times are calculated since the $\gamma$-ray trigger. See Figure 5 for a plot of the VLA observations. (EI)-(EV) indicate the five observing epochs with the VLA. References. (1) Anderson et al. (2013), (2) van der Horst (2013).

source at the location of the GRB afterglow in the reduced image, with a limiting flux of $1.1 \mathrm{mJy}(2 \sigma)$.

During Epoch I, the radio spectral index is $\beta_{\text {radio }}^{\mathrm{I}}=-2.50 \pm 0.19$, which strongly suggests that Epoch I is self-absorbed. As evident from Figure 5, at later times our 


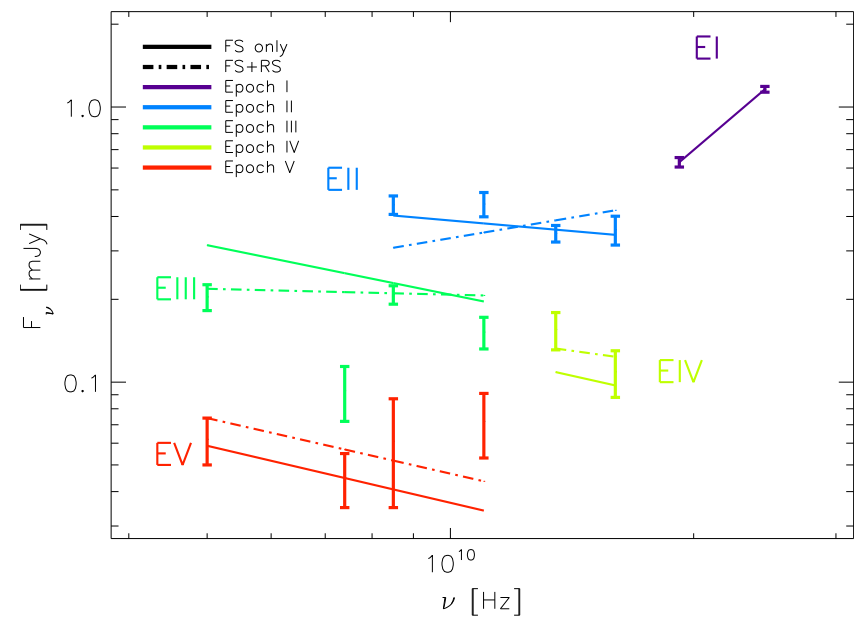

Figure 5. Compilation of VLA measurements for GRB 130907A, displaying the spectral evolution and model fits based on the forward shock only, and forward-plus-reverse shock (see Figure 6). EI through EV mark the five epochs (see Table 2).

VLA observations suggest an evolution of the radio emission toward an optically thin regime, with the spectral index progressively becoming flatter with time. In order to extract a well-sampled radio light curve from our data, we have extrapolated VLA measurements at various epochs to $15 \mathrm{GHz}$ using the best-fitting power law to the spectra at a given epoch (Table 2). For fitting purposes, we set the initial light curve slope to $\alpha=-7 / 4$, corresponding to the temporal behavior expected for a fireball expanding in a wind environment (see Section 3). With this choice, we find $\alpha=0.87 \pm 0.07$ for the late-time temporal slope at $15 \mathrm{GHz}$, and a peak time of $t_{\mathrm{r}, \mathrm{pk}}=0.38 \pm 0.03$ days (Figure 6).

\subsection{Radio-to-GeV Spectral Energy Distribution}

During Epoch I, defined by the time of the first VLA observation, we have a spectral coverage spanning $\sim 15$ orders of magnitude from radio to $\gamma$-rays (Figure 7). The spectral index in the radio band is $\beta_{\text {radio }}^{\mathrm{I}}=2.50 \pm 0.19$, which indicates that the radio emission is self-absorbed at this epoch (Section 2.4). We extrapolate the optical measurements to Epoch I using the temporal indices as derived in Section 2.3, i.e., $\alpha_{\mathrm{o}}^{\mathrm{I}} \approx 1.17-1.37$. The break in the spectrum at the intersection of the extrapolated radio and optical measurements, occurs at $\nu_{\mathrm{SA}} \approx 2 \times 10^{11} \mathrm{~Hz}$.

In the X-rays, the spectral and temporal slopes during Epoch $\mathrm{I}$ are $\beta_{\mathrm{X}}^{\mathrm{I}}=0.69 \pm 0.05$ and $\alpha_{\mathrm{X}}^{\mathrm{I}}=1.32 \pm 0.05$, respectively (see Section 2.2). The emission in the LAT energy band has a temporal index of $\alpha_{\text {LAT }}^{\mathrm{I}}=1.13 \pm 0.57$ (Tang et al. 2014), which is consistent with the X-ray one within the large uncertainties.

Interestingly, the spectrum at Epoch $\mathrm{I}$ is consistent with a single power-law component (dashed line in Figure 7) from the optical to the $\mathrm{GeV}$ range. It should be noted, however, that the low photon counts do not allow us to derive a spectral index for the $\mathrm{GeV}$ emission, thus a spectral break might be present between the X-rays and the GeV range (dotted line in Figure 7). In other words, the consistency of the X-ray and GeV (LAT) temporal indices does not require the presence of a spectral break, but the uncertainties in the LAT flux do not exclude the presence of a cooling break $\left(\nu_{\mathrm{c}}\right)$ between the X-rays and the $\mathrm{GeV}$ band. In fact, as we discuss in Section 3.8, a cooling break just above the X-ray band during Epoch I (dotted line) helps explain the late-time spectral evolution observed in X-rays. The presence of such a break would cause the LAT flux to be slightly underpredicted (by $\sim 1.2 \sigma$ ), but this could be mitigated by invoking an emergent SSC component (as suggested by Tang et al. 2014).

\section{MODELING}

\subsection{Initial Considerations}

We assume the radiation originates from synchrotron radiation of shock accelerated electrons. The electrons have a distribution described by a broken power law. The resulting synchrotron spectrum is also a set of joined power laws with breaks at the characteristic frequencies: the injection frequency, $\nu_{m}$, where the bulk of the electrons radiate; the cooling frequency, $\nu_{\mathrm{c}}$, where the cooling time of the electron radiating at this frequency is equal to the dynamical time; and the selfabsorption frequency, $\nu_{\mathrm{SA}}$, which is defined as the frequency where the optical depth for synchrotron photons becomes greater than unity for scattering on the synchrotron emitting electrons (Mészáros \& Rees 1997; Sari et al. 1998; Granot \& Sari 2002).

First, inspecting the general properties of the multiwavelength afterglow observations, this burst presents a conundrum. As we explain in what follows, based on the closure relations between the temporal and spectral indices for GRBs (see, e.g., Racusin et al. 2011), the early ( $t \lesssim 0.2$ day) $\mathrm{X}$-ray light curve slope is suggestive of a wind environment and consistent with the optical measurements. On the other hand, the radio observations are better explained in an interstellar medium (ISM) (Section 3.2). Finally, the late-time $\mathrm{X}$-ray light curve has a steep slope (Figure 1) and a strong spectral evolution (Figure 2), indicating the passage of a characteristic frequency through the X-ray band (although the steep slope of the X-ray light curve is difficult to explain in both an ISM and a wind environment).

We thus suggest that a simple external-shock model is not able to account for all the observed data. Among the multitude of extensions to the simplest model, a possible explanation is that GRB 130907A is produced by a shock initially propagating in a wind environment, which then transitions to a constant density ISM. Similar models were proposed by, e.g., Wijers (2001a), Peer \& Wijers (2006), Gendre et al. (2007), and Kamble et al. (2007). Hereafter we assume that the spectral evolution observed in the late-time X-ray afterglow is due to the passage of a characteristic frequency in band. However, we also note that the higher-than-average reddening observed in GRB 130907A suggests this burst might be a good candidate for the dust screen scenario proposed by Evans et al. (2014) to explain the spectral evolution observed in X-rays for GRB 130925A.

As the blast wave transitions from a wind to an ISM environment, roughly at the time of the X-ray break, the cooling frequency $\left(\nu_{\mathrm{c}}\right)$ sweeps through the X-rays causing the observed spectral evolution. The passage of the cooling frequency will not affect the radio light curve, which behaves simply, as in the case of an ISM.

By looking at the $15 \mathrm{GHz}$ light curve (Figure 6), we find no obvious requirement to include a reverse shock in our modeling. However, the behavior of the $5 \mathrm{GHz}$ flux seems to favor a forward-plus-reverse shock model. A better temporal coverage, particularly between 1 and 2 days since trigger at the 

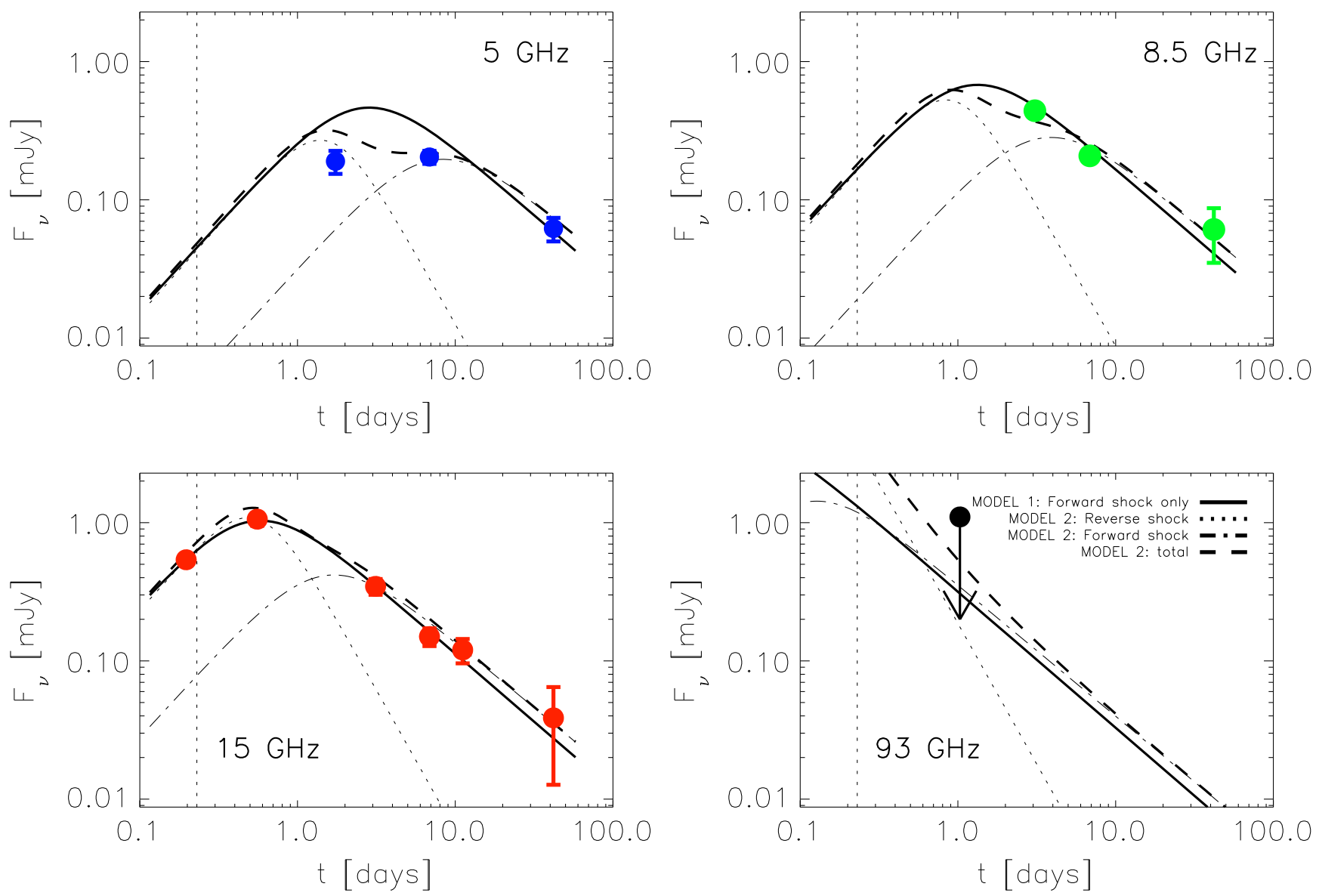

Figure 6. Radio light curves of GRB 130907A at 5, 8, 15, and $93 \mathrm{GHz}$. Continuous lines show the model consisting only of the forward shock. Dotted and dotteddashed lines represent models when both the reverse shock and the forward shock are present (their sum is plotted with dashed lines). The forward shock rising slope is $\alpha=-5 / 4$, while the reverse shock rises with $\alpha=-9 / 7$. In the ISM case, the decay slope for the forward and reverse shocks is $\alpha \approx 0.87=3(p-1) / 4$ and $\alpha=(27 p+7) / 35 \approx 2.04$, respectively. The vertical dotted line marks the transition from wind to ISM. In the wind case, the slopes of the rising part of the light curve are steeper than in the ISM case: $\alpha=-7 / 4$ (forward shock) and $\alpha=-65 / 42$ (reverse shock). Because there is only one data point in the wind regime we do not plot these cases separately. We obtain 5, 8.5, and $93 \mathrm{GHz}$ light curves by scaling the $15 \mathrm{GHz}$ light curve using the well-known synchrotron radiation scalings.

highest radio frequencies, would likely have allowed us to securely discriminate between a forward-shock-only and a forward-plus-reverse-shock scenario.

\subsection{Early-time Wind Profile}

If we assume that before the temporal break ( $t<0.23$ day) the X-ray band is below the cooling frequency and above the self-absorption and injection frequencies $\left(\nu_{m}, \nu_{\mathrm{SA}}<\nu_{\mathrm{X}}<\nu_{\mathrm{c}}\right)$, based on the spectral and temporal indices, we can estimate the nature of the interstellar matter density profile $\left(\rho \propto R^{-k}\right)$, e.g., from Sari \& Mészáros (2000): $k=4 /[1+1 /(2 \alpha-3 \beta)=$ $1.48 \pm 0.30$, which is suggestive of a wind environment before $t_{\text {break. }}{ }^{11}$ This results in a power-law index of the electron distribution of $p \approx 2.37 \pm 0.10(p=2 \beta+1)$.

The temporal evolution of the cooling frequency is $\nu_{\mathrm{c}} \propto t^{-\alpha}$, where $\alpha=-(4-3 k) /(8-2 k) \approx-0.09$, for a circumstellar density profile index $k \approx 1.5$. Thus, the cooling break is almost constant with time, similar to what was found by Perley et al. (2014) in the case of GRB 130427A.

The self-absorbed spectrum at Epoch I is a noteworthy feature of this burst, and the spectral index -2.5 is unique: The

\footnotetext{
${ }^{11}$ For a detailed treatment of the radiation from GRB afterglows in a general circumstellar density profile, see Yi et al. (2013).
}

more commonly discussed cases for synchrotron emission have a self-absorbed slope of 2 (e.g., Yost et al. 2002). The evolution of the self-absorption frequency provides another argument in favor of the wind nature of the environment closer to the explosion site. At Epoch I, $\nu_{\mathrm{SA}}^{\mathrm{I}}(0.19$ day) $\approx 200 \mathrm{GHz}$ (though this value should be considered uncertain due to the extrapolation over many orders of magnitude; see Figure 7 and Section 2.5). Epoch II is clearly not self-absorbed (Figure 5), thus $\nu_{\mathrm{SA}}^{\mathrm{II}}(3.1$ day) $\lesssim 10 \mathrm{GHz}$. In an ISM environment, $\nu_{\mathrm{SA}} \propto t^{-(3 p+2) /(2(p+4))}$, which yields a $\nu_{\mathrm{SA}}^{\mathrm{II}}(3.1$ day $) \gtrsim 20$ $\mathrm{GHz}$ for $p$ between 2 and 3. On the other hand, for a wind environment, $\nu_{\mathrm{SA}} \propto t^{-(3(p+2)) /(2(p+4))}$, which yields a selfabsorption frequency below $10 \mathrm{GHz}$ at Epoch II, in agreement with our observations.

We finally note that a more common self-absorbed spectral slope of -2 , which would be expected in the $\nu_{\text {radio }}<$ $\nu_{\mathrm{SA}}\left(<\nu_{m}<\nu_{\text {optical }}\right)$ regime, would be consistent with the observed value of $\beta_{\text {radio }}^{\mathrm{I}}=-2.50 \pm 0.19$ only at the $\approx 2.6 \sigma$ level. In this regime, because the later epochs are not selfabsorbed, $\nu_{\mathrm{SA}}$ would need to pass in the radio band by the time of Epoch II. In the ISM case, $\nu_{\mathrm{SA}} \propto t^{0}$, while in the wind case $\nu_{\mathrm{SA}} \propto t^{-3 / 5}$. Thus, our conclusion favoring an initial wind environment is not affected by the relative ordering of $\nu_{\mathrm{SA}}$ and $\nu_{m}$. 


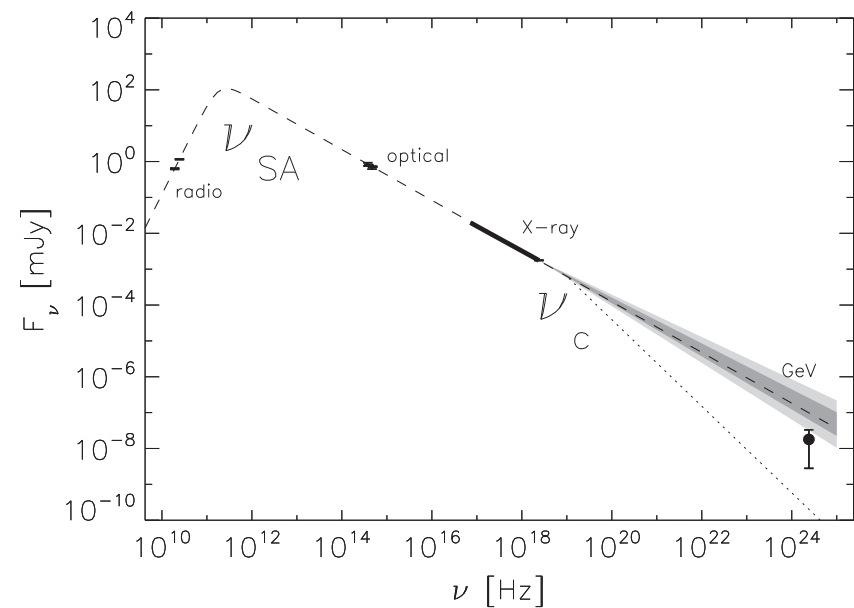

Figure 7. Spectral energy distribution of GRB 130907A during Epoch I (0.19 days after the trigger; see Table 2) from radio to $10 \mathrm{GeV}$. The broken powerlaw spectral indices are $\beta=\{-2.5,0.69\}$ for the dashed line. $\beta=1.19$ for the dotted line. $\nu_{\mathrm{SA}}$ is the self-absorption frequency and $\nu_{\mathrm{c}}$ is the cooling frequency. The dark and light shaded regions mark the $1 \sigma$ and $2 \sigma$ uncertainties, respectively, of the extrapolated X-ray spectrum.

\subsection{Spectral Energy Distribution at Epoch I}

The spectrum at Epoch I suggests a synchrotron origin for the entire spectral range (see, e.g., Kumar \& Barniol Duran 2009; Kouveliotou et al. 2013). However, in a synchrotron model one has to overcome the maximum attainable synchrotron energy condition, which might pose a problem for a synchrotron-only model (de Jager et al. 1996). A scenario for interpreting the Epoch I spectrum with a forwardshock synchrotron component is in the regime where: $\nu_{m}<\nu_{\text {radio }}<\nu_{\text {SA }}<\nu_{\text {opt }}<\nu_{\mathrm{X}}<\nu_{\mathrm{c}}<\nu_{\text {LAT }}$ (dotted line in Figure 7). Although observations are equally consistent with $\nu_{\text {LAT }}<\nu_{\text {c }}$ (dashed line in Figure 7 ), the spectral evolution observed in the late-time X-ray light curve favors a model where $\nu_{\mathrm{c}}$ lies just above the Swift/XRT range during Epoch I (Section 3.4). For the implications of an alternative inverseCompton model, see Tang et al. (2014).

\subsection{ISM Transition and Origin of the X-Ray Break}

The break observed in X-rays (Figure 1) is clearly inconsistent with a jet break because it is chromatic: It only occurs in X-rays, and the radio component does not have a break, while the optical measurements do not support it. Chromatic breaks are not uncommon in GRB afterglows (see, e.g., Panaitescu et al. 2006; Liang et al. 2007), but are not generally accompanied by spectral evolution.

No clear achromatic break can be identified in the X-ray light curve until about 20 days since the explosion. By imposing $t_{\text {jet }}>20$ day, we find $\theta_{j} \gtrsim 12^{\circ} E_{54}^{-1 / 8}\left(n / 50 \mathrm{~cm}^{-3}\right)^{1 / 8}$ $(t / 20 \text { day })^{3 / 8}((1+z) / 2.23)^{-3 / 8}$ and a beaming-corrected energy of $E_{\text {jet }} \gtrsim 3.4 \times 10^{52} \mathrm{erg}$.

To account for the observed spectral evolution, we assume the cooling frequency starts to cross the Swift/XRT band at about the same time that the shock reaches the transition from the wind environment to the ISM. This requires that the cooling break lies just above the XRT range during Epoch I, since in a $k=1.5$ medium $\nu_{\mathrm{c}}$ is almost constant with time (Section 3.2), while $\nu_{\mathrm{c}} \propto t^{-1 / 2}$ in an ISM. Indeed, the Swift/XRT data imply that $\nu_{\mathrm{c}}$ takes about two orders of magnitude in time (from $2 \times 10^{4}$ to $2 \times 10^{6}$ s) to move through approximately one order of magnitude in frequency (from 10 to $0.3 \mathrm{keV}$, the range of XRT; see Figure 2). Moreover, the observed change in spectral slope, $\Delta \beta=0.9 \pm 0.4$, is consistent with the theoretical expectations for the passage of $\nu_{\mathrm{c}}$ in band, $\Delta \beta=0.5$ (Figure 2). We note that a constant density medium might not necessarily be related to the ISM, but it might also result from the interaction of the stellar wind with the circumstellar environment. The latter may indeed be homogenized by this interaction, as suggested by several studies (e.g., van Marle et al. 2006).

Last but not least, a transition from a wind-like $(k \approx 1.5)$ medium to an ISM also allows us to explain the observed latetime slope of the radio light curve. Indeed, for a $k=1.5$ medium to yield a radio temporal slope of $\alpha \sim 0.9$ (Section 2.4), one needs an electron distribution index of $p \approx$ 1.8. ${ }^{12}$ On the other hand, in an ISM, the observed late-time radio light curve slope implies $p=4 / 3 \alpha+1=2.2 \pm 0.1$, which is consistent with the value independently derived from the early-time X-ray observations (Section 3.2).

A shortcoming of our presented model is that the X-ray temporal slope after the break is steeper than expected for a transition of the cooling frequency. In the framework of a simple synchrotron model the steepest temporal decay index is $(3 p-2) / 4$ and it does not depend on the nature of the environment. This expression for a temporal index applied to the X-ray data yields $p \approx 4.1$, which is an unusually steep value for the electron distribution index.

With the introduction of a narrow jet responsible for the $\mathrm{X}$-ray emission and a wider jet for the longer wavelength afterglow (Racusin et al. 2008; van der Horst et al. 2014), one would decouple the X-ray and optical/radio behavior. This way a break in the X-ray would be due to the narrow jet, with opening angle $\theta_{j} \sim 2^{\circ} E_{54}^{-1 / 4} A_{*,-1}^{-1 / 4}(t / 0.23 \text { day })^{1 / 4}$ $((1+z) / 2.23)^{-1 / 4}$, and an energy release corrected for beaming of $E_{\text {jet }} \approx 10^{51}\left(\theta / 2^{\circ}\right)^{2} \mathrm{erg}$. These are the opening angle and the beaming-corrected energy, respectively, if we interpret the break in X-ray as a jet break. The consequent passing of the cooling frequency would explain the spectral evolution. The radio and optical fluxes would be due to synchrotron radiation from a wider jet. This would naturally explain the late $t^{-2.57}$ behavior of the X-ray light curve with $p$ $\approx 2.57$, as the post jet-break phase flux evolves as $t^{-p}$. We consider this model as lacking solid observational evidence, but it can be substantiated for similar future bursts with better temporal coverage of the emission.

There are other possible solutions to the puzzling late-time behavior of the GRB 130907A X-ray light curve (see Section 3.4): the break in the light curve can be attributed to the end of the shallow decay (plateau) phase (Nousek et al. 2006; Liang et al. 2007) of the GRB. It is uncommon, but not impossible that the plateau phase has a temporal index of 1.3 (see Figure 4 in Grupe et al. 2013). In this case the flux before the break is related to central engine activity, and the evidence for an initial wind environment is not as compelling. The discrepancy between the late radio and X-ray temporal indices is still too large to be solved by this model.

A rather contrived setup would ascribe the radio emission to the usual synchrotron component propagating in the ISM and the X-ray flux would be given by synchrotron self-Compton

\footnotetext{
12 In order to obtain a finite energy in electrons it is normal to assume $p>2$. See, however, Panaitescu (2001) for a treatment of a $p<2$ case.
} 
radiation. The self-Compton light curves can be as steep as $t^{(9 p-11) / 8}$ for ISM to $t^{-p}$ in the wind case. It is not possible to constrain a self-Compton component from the observations, but one would need to imagine a fine-tuned interplay between the synchrotron and SSC components to explain the observed $\mathrm{X}$-ray slopes at late times.

\subsection{Transverse Size of the Jet and Scintillation}

Within the limits of our temporal coverage of the radio afterglow of GRB 130907A, no strong flux modulation due to radio scintillation is apparent. In this section, we verify a posteriori that the size of the expanding shell derived from our modeling, which assumes negligible scintillation effects, is indeed consistent with this assumption.

In a wind environment, the angle subtended by the fireball can be calculated as: $\theta_{S}=2 R_{\perp} / D_{A} \approx 5.9 \mu$ as $\left(E_{\text {iso, } 54} / A_{*,-1}\right)^{1 / 4}$ $\left(t / t_{I}\right)^{3 / 4}$, where $E_{\text {iso }}$ is the isotropic-equivalent kinetic energy of the $\mathrm{GRB}, A_{*}$ is a parameter describing the ratio of stellar mass loss to the wind velocity $\left(A_{*}=\left(\dot{M} / 10^{-5} M_{\odot} \mathrm{yr}^{-1} v_{8}^{-1}\right)\right.$, $2 R_{\perp}$ is the transverse size of the jet, and $D_{A}=D_{\mathrm{L}} /(1+z)^{2}$ is the angular diameter distance.

We use the above estimate for the size of the expanding shell to evaluate the effects of scintillation on our VLA observations (Walker 1998; Wijers 2001b). At the position of GRB 130907A (galactic coordinates $\left.(l, b) \approx\left(62^{\circ}, 55^{\circ}\right)\right)$, the upper-limit frequency for the strong scattering regime is $\nu_{0} \approx$ $7 \mathrm{GHz}$. At this frequency the source is significantly affected by scintillation if its size is smaller than $\theta_{0} \approx 4 \mu$ as. Scintillation affects the lower frequency observations more. Also, the projected size of the fireball is expected to increase with time, so later observations are less affected.

Scintillation introduces a random scatter around the "real" value of the flux. To characterize the strength of this scattering, it is customary to provide the root mean square of the fluctuations for a given frequency and a given size of the emitter. The root mean square of the scattering is given by: $m=m_{p}\left(\theta_{S} / \theta_{F}\right)^{-7 / 6}=0.08$, where $m_{p}=\left(\nu / \nu_{0}\right)^{-17 / 12}$ and $\theta_{F}=\theta_{0}\left(\nu / \nu_{0}\right)^{-1 / 2}$. Here $\nu$ is the lowest frequency in the earliest radio observation $(\nu=19.2 \mathrm{GHz})$, which is the most affected by scintillation. The low value of $m$ suggests that the Epoch I observations are not affected significantly by scintillation. The AMI observations were also carried out at an early time, thus could possibly be affected by scintillation. At 0.55 days after the trigger, at $15 \mathrm{GHz}$, similar calculations yield $m \approx 0.06$. The $5 \mathrm{GHz}$ WSRT observation at 1.74 day falls into the strong scattering regime. The theoretically expected rms caused by scintillation will be $m(5 \mathrm{GHz})=\left(\nu / \nu_{0}\right)^{17 / 30}\left(\theta_{s} / \theta\right)^{-7 / 6} \approx 0.2$. This measurement may thus be the most affected by scintillation, so we add an uncertainty of $20 \%$ in quadrature to its error. Based on our model for GRB 130907A, all other bands are expected to have insignificant distortions by scintillation.

\subsection{Emission Radius from Self-absorption}

The self-absorption frequency occurs where the synchrotron emitting electrons have the same energy that corresponds to the comoving brightness temperature $\left(T^{\prime}\right)$ of their emitted radiation. We have $\nu_{\mathrm{SA}}>\nu_{m}$ and $k T^{\prime}=\gamma_{\mathrm{SA}} m_{e} c^{2}$. Using this, we can constrain the radius of emission from (see, e.g., Shen \& Zhang 2009) $F_{\nu_{\mathrm{SA}}}\left(D_{\mathrm{L}} / R\right)^{2}=2 \gamma_{\mathrm{SA}} m_{e} c^{2} \Gamma /(1+z)^{3}$ and find $R=6.5 \times 10^{17} \mathrm{~cm} A_{*,-1}^{1 / 8} \epsilon_{B,-2}^{1 / 8}$. In deriving this result we used: $\gamma_{\mathrm{SA}}=\left(16 m_{e} c(1+z) \nu_{\mathrm{SA}} / 3 q_{e} B \Gamma\right)^{1 / 2}$ for the electron random Lorentz factor emitting at the $\nu_{\mathrm{SA}}$ frequency, $B \approx 1 \mathrm{G} \epsilon_{B,-1}^{1 / 4} A_{*,-1}^{1 / 4} E_{54}^{1 / 4}\left(t / t_{I}\right)^{-1 / 4}$ as the magnetic field strength, and $\Gamma \approx 26 A_{*,-1}^{-1 / 4} E_{54}^{1 / 4}\left(t / t_{I}\right)^{-1 / 4}$ as the bulk Lorentz factor, consistent with the calculation of Anderson et al. (2014).

We note that the above calculated radius is a factor of $\Gamma(t)$ larger than the transverse radius calculated for addressing the effects of scintillation (Section 3.5).

\subsection{Radius of the Termination Shock}

As discussed above, we interpret the break in the X-ray light curve as the transition between the wind and ISM environments. The transition radius at the time of the X-ray break $\left(t_{\mathrm{X}}\right.$, break $\approx 2 \times 10^{4} \mathrm{~s}$ ) from Panaitescu \& Kumar (2004) is $R_{t} \approx 7.7 \times 10^{17} \mathrm{~cm} E_{54}^{1 / 2} A_{*,-1}^{-1 / 2}\left(t / t_{\mathrm{X}, \text { break }}\right)^{1 / 2}$. Keeping in mind the uncertainty on the time of the X-ray break, this is broadly consistent with the radius derived from self-absorption. This radius is also close to values obtained by similar calculations (Kamble et al. 2007; Jin et al. 2009).

An approximate lower limit to the density of the ISM can be calculated by substituting the transition radius in the expression of the wind density. We find $n \gtrsim 1.2 \mathrm{~cm}^{-3}$. More accurately, from the expression of the cooling frequency (which we require to be $\sim 10 \mathrm{keV}$ at $t=0.23$ day) we have: $n \approx 50 \mathrm{~cm}^{-3} E_{54.2}^{-1 / 2} \epsilon_{B,-5}^{-3 / 2}(t / 0.23 \mathrm{day})^{-1 / 2}$, which is a reasonable value for long GRBs.

\subsection{Constraining the Micro-physical Parameters}

We assume the light curve at $15 \mathrm{GHz}$ peaks due to the passage of the self-absorption frequency through the $15 \mathrm{GHz}$ band $\left(\nu_{\mathrm{SA}}\right.$, see Figure 6$)$

Here, using the expression of the peak frequency and flux at the time of the peak in the $15 \mathrm{GHz}$ light curve (which occurs in the wind environment), we find

$$
\nu_{\mathrm{SA}}=11 \mathrm{GHz} E_{54}^{0.03} \epsilon_{e,-1}^{0.43} \epsilon_{B,-2}^{0.34} A_{*,-1}^{0.63}\left(t / t_{\mathrm{r}, \mathrm{pk}}\right)^{-1.03} \approx 15 \mathrm{GHz}
$$

and

$$
F_{\nu_{\mathrm{SA}}}=0.6 \mathrm{Jy} E_{54}^{0.82} \epsilon_{e,-1}^{1.08} \epsilon_{B,-2}^{0.61} A_{*,-1}^{0.57}\left(t / t_{\mathrm{r}, \mathrm{pk}}\right)^{-0.82} \approx 1.1 \mathrm{mJy} .
$$

The exponents are for $p=2.38$ and have a strong dependence on the value of $p$.

By assuming $\nu_{m} \lesssim 20 \mathrm{GHz}$ at the time of the first radio observation, for a wind medium (e.g., Granot \& Sari 2002) we find from the expression of $\nu_{m}$

$$
\nu_{m}=1.2 \times 10^{6} \mathrm{GHz} E_{54}^{1 / 2} \epsilon_{e,-1}^{2} \epsilon_{B,-2}^{1 / 2} \lesssim 20 \mathrm{GHz} .
$$

Roughly at the time of the temporal break in X-rays, which we associate with the transition from wind to ISM, the cooling frequency starts to sweep through the X-ray band of the XRT instrument $(0.3-10 \mathrm{keV})$. Thus we will have:

$$
\nu_{\mathrm{c}}=82 \mathrm{eVE}_{54}^{1 / 2} A_{*,-1}^{-2} \epsilon_{B,-2}^{-3 / 2} \sim 10 \mathrm{keV} .
$$

By solving Equations ((1) through (4)), we find: $A_{*} \sim 1.7$, $\epsilon_{B} \sim 1.1 \times 10^{-5}$ and $\epsilon_{e} \sim 2.0 \times 10^{-3}$, and $E \sim 1.6 \times 10^{54} \mathrm{erg}$. These values for the GRB parameters are in similar ranges as those from results from previous modelings (Panaitescu \& Kumar 2001). The value of $\epsilon_{B}$ is somewhat unusual, but not out 
of the ordinary (see, e.g., Kumar \& Barniol Duran 2010), and is consistent with no magnetic field enhancement in the forward shock, beyond the usual shock compression. Though values have to be considered with precaution as they heavily depend on the parameter $p$, they indicate that the inverseCompton cooling might be important, as suggested by Tang et al. (2014). Indeed, the power in the synchrotron selfCompton component is $Y$ times the power in the synchrotron component, with $Y$ as the Compton parameter, which for $\epsilon_{e}>\epsilon_{B}$ is $Y \approx \sqrt{\epsilon_{e} / \epsilon_{B}} \sim 13$.

Equations (1) through (4) suffer from different types of uncertainties. The value of $\nu_{m}$ is an upper limit, $\nu_{\mathrm{c}}$ is constrained within a factor of $\sim$ few to the high energy limit of XRT. Moreover, the exponents in Equations (1) and (2) have a strong dependence on the value of the parameter $p$. For these reasons, it is difficult to estimate the uncertainties for the derived parameters, and they should be treated as approximate.

\subsection{Forward-plus-reverse Shock Scenario}

From the radio data alone, there is no strong evidence for a reverse-shock component in GRB 130907A. However, the radio spectra and light curves of GRB 130907a are somewhat reminiscent of the very bright GRB 130427a (e.g., Perley et al. 2014), in which the radio data have been interpreted as a combination of a reverse and a forward shock, or a twocomponent jet. Moreover, the $5 \mathrm{GHz}$ emission of GRB $130907 \mathrm{a}$ seems to be somewhat better described by a forward-plus-reverse shock model (see Figure 6). Lastly, assuming such a component is present would alleviate the stringent constraints on the underlying physical parameters. E.g., $\nu_{m} \lesssim 20 \mathrm{GHz} \lesssim \nu_{\mathrm{SA}}$ at $t=0.19$ day can be realized with less extreme parameters (e.g., $\epsilon_{e}$ ) if we consider a reverse shock. Instead of Equation (3), we have $\nu_{m}^{\mathrm{RS}}=$ $1.1 \mathrm{GHz} E_{54}^{6 / 7} A_{*,-1}^{-5 / 14} \epsilon_{e,-1}^{2} \epsilon_{B,-2}^{1 / 2} \Gamma_{2}^{24 / 7}(t / 0.19 \text { day })^{-13 / 7}$

$\lesssim 20 \mathrm{GHz}$. Here, we assume a thin shell case for the reverse shock and $\Gamma$ is the coasting Lorentz factor of the burst. This requirement is obviously less demanding than Equation (3), and since the strongest dependence is on the $\epsilon_{e}$ parameter (disregarding the Lorentz factor, which does not enter in the forward-shock calculations), ascribing the initial rise to the reverse shock results in less extreme $\epsilon_{e}$.

The forward-reverse shock scenario introduces new parameters (e.g., temporal slope of the decaying reverse-shock component and the rising slope and peak of the forward shock) that we are unable to constrain: in Figure 6 we plot a putative reverse-plus-forward-shock model (dotted-dashed and dashed curves). We assumed an early reverse-shock component with temporal slopes fixed from theory (thin shell case in an ISM medium: $\alpha=\{-9 / 4,(27 p+7) / 35 \sim 2.38\} ; \quad$ e.g., Gao et al. 2013). For the forward shock, we fix the rising slope index to $\alpha=-5 / 4$, the peak time to 1.5 days and fit for the decaying slope. We use the ISM case here, because the only radio data in the wind regime (according to our model) is the Epoch I point. For the wind case (before the termination shock), one has steeper rising temporal indices for the reverse$(\alpha=-65 / 42)$ and forward shocks $(\alpha=-7 / 4)$. These will not change the overall properties of the components.

We apply both the forward-shock-only and reverse-plusforward-shock scenarios to the $15 \mathrm{GHz}$ light curve. Both models give a good description of the data. In an attempt to discriminate between the two, we transform the models to 5 and $8.5 \mathrm{GHz}$ to compare with observations. While the $8.5 \mathrm{GHz}$ data appears to be better described by the forward-shock-only model, the $5 \mathrm{GHz}$ measurements agree more with the twocomponent scenario (see Figure 6). The first data point at $5 \mathrm{GHz}$ is overpredicted by both scenarios and appears problematic for the forward-shock-only model, as it is $\sim 5$ standard deviations from this model.

In conclusion, within the limitations of the presented data set, we favor the forward-shock-only model (see the previous section) when compared to a forward-plus-reverse-shock model because the former is simpler and gives a similarly good description of the data. Because it is simpler, the forward-shock model also allows us to solve for the microphysical parameters (whereas the forward-plus-reverse shock model does not). However, we stress that our case study for this burst clearly indicates that a good temporal coverage at the highest radio frequencies is necessary to securely identify salient features of the reverse shock.

\section{CONCLUSION}

We have presented early radio detections of GRB 130907A with the VLA and subsequent observations as late as 42 days after the burst. Early radio observations are important for identifying potential reverse shock signatures. We complemented our radio observations with freely available data from the literature.

GRB 130907A is unusual in two respects: a chromatic steepening of the X-ray light curve at 0.23 days since the burst, which is not commonly observed, and an early-time X-ray slope that is hard to reconcile with the observed radio decay. It is a unique burst in that it has very early self-absorbed radio observations and measured spectra spanning from the radio to the $\mathrm{GeV}$ range.

To accommodate these features, we constructed a model where a blast wave propagates initially into a wind medium, then transitions into a constant density ISM. A simple forwardshock synchrotron model explains almost all of the features. We also considered a model where both reverse and forward shocks are present, but find no definitive evidence to prefer this to the simpler forward-shock-only scenario. We note, however, that the reverse and forward-shock scenario also provides a good fit, but the data is not constraining enough to argue for or against the inclusion of the reverse shock. In order to account for the spectral evolution in the X-rays, we suggest a cooling break passing in band as the shock enters the ISM.

From all the observational constraints, within the framework of the forward-shock-only-model, we derive the relevant physical parameters for this burst. The normalization of the wind density profile is $A_{*}=1.7$ in units of mass loss over wind speed. The magnetic field parameter is $\epsilon_{B} \sim 1.1 \times 10^{-5}$, while the parameter for the energy in electrons is $\epsilon_{e} \sim 2.0 \times 10^{-3}$. The total kinetic energy of this burst is comparable to the energy released in $\gamma$-rays, $E \sim 1.6 \times 10^{54} \mathrm{erg}$. These parameters have a strong dependence on the power-law index of the electron distribution, which we set to $p \approx 2.38$.

With the isotropic-equivalent energy in excess of $10^{54} \mathrm{erg}$, and beaming-corrected energy in excess of $3 \times 10^{52} \mathrm{erg}$, GRB $130907 \mathrm{~A}$ is part of the hyper-energetic bursts. If GRB 130907 has a double-jet structure, the beaming-corrected energy of the narrow component reduces to the more typical value of $10^{51} \mathrm{erg}$. The properties of this burst can be compared to the broader sample of LAT-detected GRBs or bursts with an identified wind-ISM transition. 
Comparing our derived parameters with those of the LATdetected sample (see Table 3 in Racusin et al. 2011), we find this burst typical in many respects. In terms of both kinetic $\left(1.6 \times 10^{54} \mathrm{erg}\right)$ and radiated $\left(3 \times 10^{54} \mathrm{erg}\right)$ isotropicequivalent energy, this is an average GRB. Furthermore, in terms of energy conversion efficiency $\left(E_{\gamma} /\left(E_{\gamma}+E_{K}\right) \sim 65 \%\right)$, this burst is in the middle of the distribution of LATdetected GRBs.

The value of the termination shock radius $\left(\sim 7.7 \times 10^{17} \mathrm{~cm}\right)$ is consistent with the ones derived from similar wind-ISM transition modeling. However, they all suffer from the inconsistency already noted in the literature (e.g., Jin et al. 2009) between numerical models of Wolf-Rayet (WR) stars' termination shock radii $\left(\gtrsim 3 \times 10^{18} \mathrm{~cm}\right)$ and GRBdeduced values. This can be mitigated, e.g., if the stellar wind is anisotropic (Eldridge 2007; van Marle et al. 2008), if the WR wind is weak, or if it resides in a high-density or high-pressure ISM (van Marle et al. 2006).

The parameters obtained for GRB 130907A, perhaps with the exception of the density parameter, are in the same range among the GRBs with claimed wind-ISM transition. The $A_{*}$ parameter of this burst is larger by $\sim 2$ orders of magnitude compared to other GRBs. For similar total isotropic energy $\left(\sim 10^{54} \mathrm{erg}\right)$ GRB 081109 (Jin et al. 2009) has $\epsilon_{e}$ and $\epsilon_{B}$ parameters that are larger by an order of magnitude, while the wind density parameter $\left(A_{*}\right)$ is two orders of magnitude lower. GRB 081109 has a low efficiency $(\sim 1 \%)$ compared to GRB 130907A (65\%). In case of GRB 050319 (Kamble et al. 2007), $\epsilon_{e}$ is the same order of magnitude as in our case, $\epsilon_{B}$ is significantly (3-4 orders of magnitude) higher, and $A_{*}$ is 2-3 orders of magnitude lower.

In conclusion, GRB 130907A poses intriguing challenges in the modeling of its multi-wavelength observations, since simple ISM or wind density profiles fail to account for the entire set of observations. Invoking the wind-ISM transition is a natural extension of the wind-only scenario, and one would expect to have such a transition in all wind density profiles as the shock reaches the ISM surrounding the progenitor star. This burst adds to the small number of GRBs showing this transition.

We thank Phil Evans and Bing Zhang for valuable discussions, and the anonymous referee for useful comments. P.V. acknowledges support from Fermi grant NNM11AA01A and OTKA NN 111016 grant. A.C. acknowledges partial support from the NASA-Swift GI program via grants 13SWIFT13-0030 and 14-SWIFT14-0024. Support for S.B.C. was provided by NASA through the Fermi grant NNH13ZDA001N. This work made use of data supplied by the UK Swift Science Data Centre at the University of Leicester.

\section{REFERENCES}

Akerlof, C., Balsano, R., Barthelmy, S., et al. 1999, Natur, 398, 400 Anderson, G. E., Fender, R. P., Staley, T. D., \& Rowlinson, B. A. 2013, GCN, 15211,1

Anderson, G. E., Fender, R. P., Staley, T. D., et al. 2014, GCN, 16595, 1

Anderson, G. E., van der Horst, A. J., Staley, T. D., et al. 2014, MNRAS, 440, 2059

Beuermann, K., Hessman, F. V., Reinsch, K., et al. 1999, A\&A, 352, L26

Butler, N., Watson, A. M., Kutyrev, A., et al. 2013a, GCN, 15208, 1

Butler, N., Watson, A. M., Kutyrev, A., et al. 2013b, GCN, 15209, 1

Butler, N., Watson, A. M., Kutyrev, A., et al. 2013c, GCN, 15223, 1

Castro-Tirado, A. J., Bremer, M., \& Winters, J.-M. 2013, GCN, 14689, 1
Cenko, S. B., Frail, D. A., Harrison, F. A., et al. 2011, ApJ, 732, 29

Chandra, P., \& Frail, D. A. 2012, ApJ, 746, 156

Corsi, A., Perley, D. A., \& Cenko, S. B. 2013, GCN, 14990, 1

Corsi, A., Piro, L., Kuulkers, E., et al. 2005, A\&A, 438, 829

Corsi, A. 2013, GCN, 15200, 1

Corsi, A. 2014a, GCN, 17070, 1

Corsi, A. 2014b, GCN, 17019, 1

Corsi, A. 2014c, GCN, 16516, 1

de Jager, O. C., Harding, A. K., Michelson, P. F., et al. 1996, ApJ, 457, 253 de Ugarte Postigo, A., Xu, D., Malesani, D., et al. 2013, GCN, 15187, 1 Eldridge, J. J. 2007, MNRAS, 377, L29

Evans, P. A., Beardmore, A. P., Page, K. L., et al. 2007, A\&A, 469, 379

Evans, P. A., Beardmore, A. P., Page, K. L., et al. 2009, MNRAS, 397, 1177

Evans, P. A., Willingale, R., Osborne, J. P., et al. 2010, A\&A, 519, A102

Evans, P. A., Willingale, R., Osborne, J. P., et al. 2014, MNRAS, 444, 250

Fong, W. 2014, GCN, 16777, 1

Fong, W., Zauderer, A., \& Berger, E. 2012a, GCN, 13587, 1

Fong, W., Zauderer, B. A., \& Berger, E. 2011, GCN, 12571, 1

Fong, W., Zauderer, B. A., \& Berger, E. 2012b, GCN, 14126, 1

Fong, W., Zauderer, B. A., \& Berger, E. 2013a, GCN, 14751, 1

Fong, W., Zauderer, B. A., \& Berger, E. 2013b, GCN, 15122, 1

Fong, W., Zauderer, B. A., \& Berger, E. 2013c, GCN, 15227, 1

Fong, W., Zauderer, B. A., \& Berger, E. 2013d, GCN, 15626, 1

Gao, H., Lei, W.-H., Zou, Y.-C., Wu, X.-F., \& Zhang, B. 2013, NewAR, 57, 141

Gehrels, N., Chincarini, G., Giommi, P., et al. 2004, ApJ, 611, 1005

Gendre, B., Galli, A., Corsi, A., et al. 2007, A\&A, 462, 565

Golenetskii, S., Aptekar, R., Frederiks, D., et al. 2013, GCN, 15203, 1

Gorbovskoy, E., Lipunov, V., Denisenko, D., et al. 2013a, GCN, 15220, 1

Gorbovskoy, E., Lipunov, V., Kornilov, V., et al. 2013b, GCN, 15184, 1

Gordon, K. D., Clayton, G. C., Misselt, K. A., Landolt, A. U., \& Wolff, M. J. 2003, ApJ, 594, 279

Granot, J., \& Sari, R. 2002, ApJ, 568, 820

Grupe, D., Nousek, J. A., Veres, P., Zhang, B.-B., \& Gehrels, N. 2013, ApJS, 209, 20

Hancock, P., Murphy, T., Gaensler, B., et al. 2012, GCN, 13180, 1

Hancock, P. J., Murphy, T., Gaensler, B., \& Zauderer, A. 2011, GCN, 12804, 1

Hentunen, V.-P., Nissinen, M., Vilokki, H., \& Salmi, T. 2013, GCN, 15201, 1

Horesh, A., Carpenter, J., Corsi, A., et al. 2011, GCN, 12710, 1

Jin, Z. P., Xu, D., Covino, S., et al. 2009, MNRAS, 400, 1829

Kamble, A., Resmi, L., \& Misra, K. 2007, ApJL, 664, L5

Kouveliotou, C., Granot, J., Racusin, J. L., et al. 2013, ApJL, 779, L1

Kulkarni, S. R., Djorgovski, S. G., Odewahn, S. C., et al. 1999, Natur, 398, 389

Kumar, P., \& Barniol Duran, R. 2009, MNRAS, 400, L75

Kumar, P., \& Barniol Duran, R. 2010, MNRAS, 409, 226

Laskar, T., Zauderer, A., \& Berger, E. 2012a, GCN, 13547, 1

Laskar, T., Zauderer, A., \& Berger, E. 2012b, GCN, 13578, 1

Laskar, T., Zauderer, A., \& Berger, E. 2012c, GCN, 13903, 1

Laskar, T., Berger, E., Zauderer, B. A., et al. 2013a, ApJ, 776, 199

Laskar, T., Zauderer, B. A., Berger, E., et al. 2013b, GCN, 14817, 1

Laskar, T., Zauderer, B. A., Berger, E., et al. 2014a, GCN, 15985, 1

Laskar, T., Zauderer, B. A., Berger, E., et al. 2014b, GCN, 16283, 1

Lee, W. H., Butler, N., Watson, A. M., et al. 2013, GCN, 15192, 1

Liang, E.-W., Zhang, B.-B., \& Zhang, B. 2007, ApJ, 670, 565

Melandri, A., Mundell, C. G., Kobayashi, S., et al. 2008, ApJ, 686, 1209

Mészáros, P., \& Rees, M. J. 1997, ApJ, 476, 232

Mészáros, P., \& Rees, M. J. 1999, MNRAS, 306, L39

Michalowski, M., Xu, D., Stevens, J., et al. 2011, GCN, 12422, 1

Nousek, J. A., Kouveliotou, C., Grupe, D., et al. 2006, ApJ, 642, 389

Ofek, E. O., Frail, D. A., Breslauer, B., et al. 2011, ApJ, 740, 65

Oates, S. R., \& Page, M. J. 2013, GCN, 15195, 1

Page, M. J., Beardmore, A. P., Burrows, D. N., et al. 2013, GCN, 15183, 1

Panaitescu, A. 2001, ApJ, 556, 1002

Panaitescu, A., \& Kumar, P. 2001, ApJL, 560, L49

Panaitescu, A., \& Kumar, P. 2004, MNRAS, 353, 511

Panaitescu, A., Mészáros, P., Burrows, D., et al. 2006, MNRAS, 369, 2059

Peer, A., \& Wijers, R. A. M. J. 2006, ApJ, 643, 1036

Perley, D. A. 2013a, GCN, 14387, 1

Perley, D. A. 2013b, GCN, 14494, 1

Perley, D. A. 2013c, GCN, 15478, 1

Perley, D. A. 2014, GCN, 16122, 1

Perley, D. A., Alatalo, K., \& Horesh, A. 2012, GCN, 13175, 1

Perley, D. A., \& Kasliwal, M. 2013, GCN, 14979, 1

Perley, D. A., \& Keating, G. 2013, GCN, 14210, 1

Perley, D. A., Cenko, S. B., Corsi, A., et al. 2014, ApJ, 781, 37

Perley, R., Napier, P., Jackson, J., et al. 2009, IEEEP, 97, 1448 
Pozanenko, A., Volnova, A., Hafizov, B., \& Burhonov, O. 2013, GCN, 15240, 1 Racusin, J. L., Karpov, S. V., Sokolowski, M., et al. 2008, Nature, 455, 183 Racusin, J. L., Oates, S. R., Schady, P., et al. 2011, ApJ, 738, 138

Sari, R., \& Mészáros, P. 2000, ApJL, 535, L33

Sari, R., \& Piran, T. 1999, ApJL, 517, L109

Sari, R., Piran, T., \& Narayan, R. 1998, ApJL, 497, L17

Schady, P., Page, M. J., Oates, S. R., et al. 2010, MNRAS, 401, 2773

Schlafly, E. F., \& Finkbeiner, D. P. 2011, ApJ, 737, 103

Schmidl, S., Kann, D. A., Klose, S., et al. 2013, GCN, 15194, 1

Shen, R.-F., \& Zhang, B. 2009, MNRAS, 398, 1936

Tang, Q.-W., Tam, P.-H. T., \& Wang, X.-Y. 2014, ApJ, 788, 156

Tanvir, N. R., Levan, A. J., Wiersema, K., \& Cucchiara, A. 2014, GCN, 15961, 1

Trotter, A., Haislip, J., LaCluyze, A., et al. 2013a, GCN, 15191, 1

Trotter, A., Reichart, D., Haislip, J., et al. 2013b, GCN, 15193, 1

Urata, Y., Huang, K., Takahashi, S., et al. 2014, ApJ, 789, 146

van der Horst, A. J. 2013, GCN, 15207, 1

van der Horst, A. J., et al. 2014, arXiv:1404.1945

van Marle, A. J., Langer, N., Achterberg, A., \& García-Segura, G. 2006, A\&A, 460,105

van Marle, A. J., Langer, N., Yoon, S.-C., \& García-Segura, G. 2008, A\&A, 478,769
Vianello, G., Kocevski, D., Racusin, J., \& Connaughton, V. 2013, GCN, 15196, 1

Walker, M. A. 1998, MNRAS, 294, 307

Wijers, R. A. M. J. 2001a, in Gamma-ray Bursts in the Afterglow Era, ed. E. Costa, F. Frontera, \& J. Hjorth (Berlin: Springer), 306

Wijers, R. A. M. J. 2001b, MNRAS, 321, 176

Yi, S.-X., Wu, X.-F., \& Dai, Z.-G. 2013, ApJ, 776, 120

Yost, S. A., Frail, D. A., Harrison, F. A., et al. 2002, ApJ, 577, 155

Zauderer, A., \& Berger, E. 2011, GCN, 12496, 1

Zauderer, A., \& Berger, E. 2012a, GCN, 12895, 1

Zauderer, A., \& Berger, E. 2012b, GCN, 13336, 1

Zauderer, A., Berger, E., \& Frail, D. 2011, GCN, 12436, 1

Zauderer, A., Berger, E., \& Laskar, T. 2012a, GCN, 13813, 1

Zauderer, A., Laskar, T., \& Berger, E. 2012b, GCN, 13231, 1

Zauderer, B. A., Berger, E., Chakraborti, S., \& Soderberg, A. 2013a, GCN, 14480, 1

Zauderer, B. A., Berger, E., \& Laskar, T. 2013b, GCN, 14172, 1

Zauderer, B. A., Fong, W., \& Berger, E. 2012c, GCN, 13010, 1

Zauderer, B. A., Fong, W., \& Berger, E. 2014a, GCN, 16593, 1

Zauderer, B. A., Fong, W., Berger, E., \& Laskar, T. 2013c, GCN, 14863, 1

Zauderer, B. A., Laskar, T., \& Berger, E. 2014b, GCN, 15931, 1

Zhang, B., \& Kobayashi, S. 2005, ApJ, 628, 315 